\title{
MICROVASCULAR DECOMPRESSION TREATMENT OF TRIGEMINAL NEURALGIA
}

\author{
Nebi Yılmaz', Gökhan Akdemir' ${ }^{2}$ Yavuz Akbay ${ }^{3}$, Yusuf Aslantürk', Fikret Ergüngör ${ }^{2}$
}

Yüzüncü Y1l University, Faculty of Medicine, Department of Neurosurgery ${ }^{1}$ and Ankara Numune Education and Research Hospital, Neurosurgical Clinic ${ }^{2}$, Kırıkkale State Hospital ${ }^{3}$ Muş State Hospital $1^{4}$, Turkey.

\begin{abstract}
Aim: To evaluated 20 trigeminal neuralgia patients who were resistant to medical treatments underwent surgery between years 1997-2000 in our clinic.

Methods: The surgical procedure was microvascular decompression with lateral suboccipital approach. Surgical results and complications were compared to other techniques with review of the literature. Twenty patients with a mean age of 55 underwent surgery, 12(12\%) of them were female and $8(40 \%)$ of them were male.

Results: Pain was on the left side in $11(55 \%)$ and on the right side in 9(45\%) patients. Diagnosis of the trigeminal neuralgia was based on the followings: Response to carbamazepine treatment, analysis of the pain with a detailed history, neurological examination and radiological evaluation. 8 patients had a history of hypertension and none of the patients in entire group had a known diagnosis of multiple sclerosis. Pain was experienced mostly on V1,V2 and V2,V3. Cranial magnetic resonance imaging (MRI) was performed to all patients. The etiological factors were estimated as idiopathic or vascular compression in 19, and epidermoid tumor in 1 patient. Patients were operated in semisitting position with a mayfield and lateral suboccipital surgical approach was chosen. Microvascular decompression was applied in 15 patients by using teflon felt and in 4 patients by using goretex. The epidermoid tumor was evacuated in one patient. Superior cerebellar artery was found as the commonest artery that caused entry zone nerve compression. During the postoperative period of 4 months to 3.5 years the $15(\% 75)$ of the patients were pain-free without any medical treatment; $2(\% 10)$ patients were pain-free on welltolerated medication and mild-pain without medication; $2(\% 10)$ patients had no pain relief; and $1(\% 5)$ patient was pain-free after the operation for more than 1 month followed by the recurrence of pain requiring medical or surgical therapy. Microvascular decompression technique has been suggested superior to alternative methods for its safety and efficacy without recurrence.

Conclusion: Our results support the notion that microvascular decompression is the surgical treatment of choice for patients with trigeminal neuralgia who are younger than 70 year-old, have no risk for anesthesia and who are resistant to medical treatments.
\end{abstract}

Key words: Trigeminal neuralgia, pain, microvascular decopression, trigeminal nerve

Eur J Gen Med 2005; 2(3):114-119

\section{INTRODUCTION}

Trigeminal neuralgia is a paroxysmal disorder that is characterized by severe pain attacks spreading over the location where one or more trigeminal nerve innervates. Its' etiology remains unknown. The disorder is more common in women than in men and mostly affects hypertensive patients and patients older than 50 (1). Trigeminal neuralgia can be treated both medically and surgically. If carbamazepine, baclophen and phenitoine are not effective as medical treatments, surgical procedures are applied. There may be no visible neurological deficits in the clinical examination of the patients. The characteristics of the pain and response to carbamazepine are important in the diagnosis of the illness (2) In the latest 
Table 1. Factors initiating the pain

\begin{tabular}{lccc}
\hline Factor initiating the pain & I. Factor & II Factor & III. Factor \\
\hline Touch & 10 & 6 & 3 \\
Eating and drinking & 5 & 6 & 6 \\
Talking and laughing & 4 & 5 & 7 \\
Unprompted & 1 & 2 & 3 \\
With wind & 0 & 1 & 1 \\
\hline
\end{tabular}

years, MRI (magnetic resonance imaging) and MRA (magnetic resonance angiography) made it possible to exhibit the relationship between the trigeminal nerve and vascular structures that cause compression. (3). The understanding that trigeminal neuralgia is caused by trigeminal nerve root entry zone subject to compression caused by nerve structures, tumor or other reasons is accepted. Dandy reported that, the main reason of compression is anterior inferior cerebellar artery and he was the frontier of the surgical treatment of trigeminal neuralgia. Peter Jannetta was the first neurosurgeon to apply the operating microscope to the problem of trigeminal neuralgia and devised a technique for nondestructive microvascular decompression of the nerve(4,5,6). In surgery, death can occur due to air emboli, vascular injuries and infarct. Hematoma in the postoperative operation area and function disorders in VII and VIIIth cranial nerves can be observed(7,8).

\section{MATERIAL AND METHODS}

Twenty patients with the diagnosis of trigeminal neuralgia and non responsive to medical treatment were operated in our clinic between the years 1997 and 2000. Of the operated 20 patients, 12 patients were (\% $60)$ women, 8 patients were $(\% 40)$ men and the youngest patient was 37 years old while the oldest was 70 years old. The average age of the patients was 55. Although there was no significant characteristic in the past histories of the cases, hypertension captured attention. Eight patients had a past history of hypertension and only four of them were receiving regular anti-hypertensive medicine. All of the patients had used carbamazepine and the average period of using carbamazepine is 12 years. The longest duration of carbamazepine usage is 25 years and the shortest duration is about 4 years. Only three of the patients were treated by doing injection to the face and 8 patients were applied dental procedures due to the pain. All of the patients admitted with facial pain. Out of 20 patients, the pain was reported to be on the left side of the face in 11 patients $(\% 55)$, in 9 patients $(\% 45)$ on the right side of the face. The pain was typical in 12 of the patients and atypical in 8 of the patients. The patients defined the pain as explosive, sharp, severe, similar to lightning and knife cut. The patients had no complaints between the pain attacks. The average duration of the typical pain was 1 minute, the shortest pain duration was 5-10 seconds and the longest was 2 minutes. It was observed that in patients with atypical pain the duration of the pain was longer, the duration between the attacks was shorter and resistance to carbamazepine treatment was higher. It was observed that patients with atypical pain were using higher doses of carbamazepine. Although all patients had both day and night pain, there was a significant difference in the frequencies of day and night pain. Except two patients with atypical trigeminal neuralgia, remaining 18 patients reported that night pain was rare. Pain can be activated by touching, eating, drinking, talking, wind and with no significant stimuli (Table 1). The most frequent reason in activating the pain was observed as touching. When the patients were neurologically examined, there was no finding except for hypoesthesia. The cornea reflex was found to be normal in all of the patients. The distribution of the trigeminal nerve in the face was observed in V1 and V2 in 6 cases $(\% 30)$ and in V2 and V3 again in 6 cases (\% 30). Later on, in V1 and V2 with 4 cases $(\% 20)$ in V2 with 3 cases $(\% 15)$ and in V3 with 1 case $(\% 5)$.

Computer Tomography(CT) and Magnetic Resonance; MRI was applied to all patients; cranial CT was applied to 4 patients and temporal CT to one patient. With cranial MRI, alterations, consistent with cortical atrophy was observed in three patients. In one patient, there was a finding that may be compatible with pathological change and angiography was applied to this patient. The result of the angiography was evaluated as normal. The etiology of trigeminal neuralgia was evaluated as idiopathic and due to 
Table 2. The frequency of vascular structures that lead to compression

\begin{tabular}{lcc}
\hline Vascular structures that lead to compression & number of patient & Percentage \\
\hline Superior cerebellar artery & 8 & $40 \%$ \\
Anterior inferior cerebellar artery & 5 & $25 \%$ \\
Venous structures & 4 & $20 \%$ \\
Posterior inferior cerebellar artery & 2 & $10 \%$ \\
Tumor (epidermoid) & 1 & $5 \%$ \\
\hline
\end{tabular}

vascular compression in 19 patients and due to an epidermoid (tumor) in one patient. None of the patients had multiple sclerosis. In addition, pontocerebellar infarct, dental illness and herpetic neuralgia were not detected in the patients.

Twenty patients with trigeminal neuralgia and non responsive to medical treatment were operated by using microvascular decompression technique in our clinic. Three patients had percutaneous rhysotomy and one patient had peripheral neurectomy previously, but patients did not benefit from these interventions. There was no pathological condition that would prevent the application of general anesthesia. Carpamazepine was applied $600 \mathrm{mg} /$ day postoperatively. Later on, this dosage was stopped in patients with no pain and in patients who displayed significant recovery. Therefore, carbamazepine usage was arranged according to the postoperative pain. All of the patients received steroid (methylprednisolone) treatment both before and after the operation.

Cranial nerve manipulation was avoided during microvascular surgery and unnecessary cerebellar ecartation was not applied. Transversal sinus was lower than place in a case. Trigeminal nerve was reached via tentorium in this patient. Except the patient with tumor, vascular structures related with root entry zone of the trigeminal nerve and compresses the area was clearly visible in 19 cases. The rates of vascular structures that lead to compression are shown in Table 2. It was found that superior cerebellar artery is the most frequent cause of compression in the patients. (In 8 patients, 40\%) Except vascular compression in only one case, the etiology of trigeminal neuralgia was found to be an epidermoid tumor.

These vascular structures were repositioned by carefully pulling out from the trigeminal nerve. Later on, the implant was placed between the trigeminal nerves and vascular structures. As the implant Teflon felt was used in 15 patients and gorotex in 5 patients. The stability of this new anatomic position was checked by applying Valsalva maneuver with the control of anesthesiologist. The stability of this condition was reobserved after the pulling of cerebellar achartory. In the patient with epidermoid tumor, the tumor was totally drained and compression of the root entry zone was eliminated.

There were no intra-operative complications in the patients and mortality did not occur. After the operation, some of the patients reported headache and nausea problems. All these problems recovered 45 days after the operation, but decreased headache continued in some patients. CSF leakage, infection, hearing loss, hydrocephaly, cerebellar hematoma and infarct were not observed in any of the patients. Facial paralysis was observed in five patient in the early postoperative period, but all except one had recovered. We have conducted facial nerve function according to House and Brakeman's clinical classification in the early postoperative period. All of the grade2 facial paralysis patients and one out of 3 grade-3 patients were recovered. In one of the patients facial paralysis regressed to grade 2 but in one patient facial paralysis continued as grade 4 .

\section{DISCUSSION}

Trigeminal neuralgia is a thoroughly researched disorder with various characteristics that can be treated with medication and surgery. The doctor should explain the advantages and disadvantages of the operation to the patient beforehand and should make sure that the patient has understood the procedure. He should give information about other alternative treatments and should suggest his own treatment preference to the patient. All writers now accept compression of the trigeminal nerve on the root entry zone, which was previously accepted as a theory as the main reason. Therefore, microvascular decompression surgery gained emphasis in the surgical treatment of trigeminal neuralgia $(9,10,11,12)$.

Trigeminal neuralgia is divided into two 
Table 3. Classification of UCSF (University of California at San Francisco) was used in the evaluation of the results of the microvascular decompression treatment.

\begin{tabular}{|c|c|c|c|}
\hline Results & Definition & $n$ & $\%$ \\
\hline Perfect & Patients with no pain and do not need treatment & 15 & $(75 \%)$ \\
\hline Good & Medium level of pain with/or without medical treatment & 2 & $(10 \%)$ \\
\hline Painless & Painless period for more than a month following surgery & 1 & $(1 \%)$ \\
\hline Recurrent & or recurrent pain that requires medical treatment. & & \\
\hline Poor & $\begin{array}{l}\text { Non-recovery in the pain following the operation } \\
\text { or a very little ease in the pain }\end{array}$ & 2 & $(10 \%)$ \\
\hline
\end{tabular}

subgroups as typical and atypical according to the characteristic of the pain and the condition of hypoesthesia. Generally, it starts as typical trigeminal neuralgia and if untreated may progress atypically and there may be cases with atypical initiation $(1,13)$. In this study, three cases had an atypical initiation and 5 cases had progresses from typical to atypical trigeminal neuralgia. It was proposed that, carbamazepine response was low in patients with atypical trigeminal neuralgia. In this study the fast elevation of the dosage in the medical treatment in 8 patients with atypical trigeminal neuralgia captured attention.

Recurrence was more frequent in patients with atypical trigeminal neuralgia following microvascular decompression surgery and vascular decompression being more frequently originated from the blood veins in atypical trigeminal neuralgia was accepted as the reason (14). Burchiel et al. found that, nerve compression is elevated in patients with atypical trigeminal neuralgia(12). In our study, we found that, vascular decompression was more significant in eight patients operated in our clinic with atypical trigeminal neuralgia than patients with typical trigeminal neuralgia Trigeminal neuralgia is more common in middle and advanced ages. Jannetta stated that, the relationship between trigeminal neuralgia and arteriosclerosis is obvious and proposed that atherosclerotic elongation produced new loops (7). Again, the finding that trigeminal neuralgia is more common in middle and advanced age supports its relationship with arteriosclerosis Briefly, it can be summarized as, the move of atherosclerotic veins especially the horizontal loop of superior cerebellar artery to pons without widening laterally. Therefore, the stretched vascular structure becomes adequate for nerve compression by changing position. Trigeminal neuralgia is more frequent in women than men. In our cases this ratio was found as $1.5 / 1$. The reason of trigeminal neuralgia being more frequent in women is unknown, but there are some opinions on the issue. Presence of smaller posterior fossa in women was thought to be an explanation but this was not proved. Studies on the estrogen hormone are still scarce. The pain may be localized in one or more branches of the trigeminal nerve. In many articles, it was concluded that the most frequently effected nerve branches are V2-V3 or V1-V2. There was no difference in terms of the side of the face in patients. The rate of bilateral trigeminal neuralgia was reported as approximately $2 \%$. Bilateral trigeminal neuralgia was especially reported in patients with multiple sclerosis (15).

There is a consensus on initiating carbamazepine treatment in patients diagnosed with trigeminal neuralgia. If the patient does not respond, carbamazepine and baclophen combination treatment had been suggested. If this treatment modality fails either surgical treatment or dilantin treatment can be preferred. Medical treatment except carpamazepine was not applied to patients operated in our clinic. Sjaastad, conducted research on patients who did not respond to medical treatment in order to determine the next step of the treatment and concluded that, the next step in the treatment is depended on the etiology of the trigeminal neuralgia.

Writers have suggested that the preferred treatment should be retromastoid microvascular decompression if the reason of pain is hypothesized as vascular compression or as idiopathic for patients younger than 65 years and if there is no important surgical risk. If recurrence occurs after this surgical intervention than microvascular decompression operation should be repeated. If this intervention fails, PRGR(Percutaneous Retrogasserian Glycerol Rhizotomy) and PRRR (Percutaneous Retrogasserian Radiofrequency Rhizotomy) should be preferred. Microvascular decompression treatment was applied to 19 patients in our clinic. Full recovery was observed in 15 (75\%) 
of these patients(Table 3 ). Jannetta reported a full recovery in the long-term follow-up of $449(\% 73)$ patients out of 618 cases treated with microvascular decompression (8).

It was agreed that microvascular decompression treatment should not be used in patients with multiple sclerosis. Because, the etiology of pain in these patients is due to demyelinised plaques in the trigeminal nerve root entry zone. The suggested treatment in multiple sclerosis patients is PRGR or PRRR. In recurrences, it was suggested that these treatments should be repeated (15). None of the cases out of 20 had multiple sclerosis in our study. If the reason of trigeminal neuralgia is a tumor, the tumor should be removed with surgical intervention. If the tumor cannot be removed or if the pain continues after the release of the tumor, then PRGR or PRRR treatment should be performed. One trigeminal neuralgia case caused by a tumor was operated in our clinic. The tumor was totally removed, macroscopic and microscopically it was evaluated as an epidermoid tumor. The post-operative pain was totally released and there was no recurrence during the 2 months follow-up period.

Surgical experience and a high-quality anesthesia can reduce the problems in microvascular decompression surgery. Mortality, intracranial hemorrhage and infarct were not observed in the twenty patients who had microvascular decompression treatment in our clinic. Cranial nerve defect did not occur except for the facial nerve. Mortality was found in $2(0.3 \%)$ cases out of 618 cases that had microvascular decompression surgery by Jannetta and in 3 months-4 years follow up recurrence rate was found as $25 \%$.(7) In a study, Young et al. treated 19 patients with trigeminal neuralgia by using gamma knife radiosurgery technique and reported that the results were successful when compared with other treatment modalities. Again, in another study conducted with 51 patients, Young et al. applied maximum 70 Gy radiosurgery to the trigeminal nerve root near to pons, nerve root was localized with streotactic MRI and a total recovery in pain was reported in \%74.5 of the cases (16). Following these studies it was concluded that, due to its dependability and minimal invasiveness gamma knife surgery is feasible in the treatment of trigeminal neuralgia (17).

Microvascular decompression treatment is being considered in other neurovascular compression syndromes such as, hemifacial spasm, genuculate neuralgia, vertigo and torticollis due to the 11 th cranial nerve. Jannetta applied microvascular decompression treatment to 14 patients with geniculate neuralgia. By applying retromastoid craniectomy in the surgery, microvascular decompression of the nevrus impedus was accomplished and they obtained positive results in 10 of these patients(8).

Waidhauser et al. evaluated atypical trigeminal neuralgia patients with ganglion gasserinine test stimulation for stabile electrode implant. They have started the study by proposing that the application of destructive procedures in atypical patients generally lead to the worsening of the patients' clinic. In their study, they have applied ganglion gasserinine percutaneous test stimulation to 149 patients in hospital conditions. In $54 \%$ cases (n:81) pain was released during test applications thus stable electrodes were applied to these patients. Therefore, they have accepted the ganglion test stimulation as a key for a good therapeutic result in patients with trigeminal neuralgia(18).

Trigeminal neuralgia cases are generally in middle or old ages and mostly women. It was observed that hypertension is significantly more frequent in patients with trigeminal neuralgia and this was explained but the reason of female predominance couldn't be explained. Number of cases with left side pain was higher but in the literature there are series in which both left and right sided pain occurs and no significant difference was reported. The pain was most frequently found in V1-V2 (30\%) and V2-V3 (30\%) areas. A comprehensible conclusion related with the relationship between vascular structures and trigeminal nerve could not be made because the quality and teslas of the MRI in patients were low. In this regard, it was clear in the literature that good quality preoperative MRI and MRA would be useful $(19,20)$. Mortality was not seen in any of the patients. Anesthesia, operation rooms and intensive care units with modern devices and experienced teams had positive effect in mortality and morbidity.

As a result, in trigeminal neuralgia patients the primary choice treatment is microvascular decompression when the patient does not respond to medical treatment and there is no risk in taking general anesthesia. 


\section{REFERENCES}

1. Ecker AD. The cause of the trigeminal neuralgia. Med Hypotheses 2004;62:520

2. Abdeen K, Kato Y. Kiya N, Yoshida K, Kanno T. Neuroendoscopy in microvacular decompression for trigeminal neuralgia and hemifacial spasm technical note. Neurol Res 2000;22:522-6

3. Kamishima K, Umehara F, Kasio $\mathrm{N}$ et al. Magnetic resonance tomographic angiography diagnostic value in trigeminal neuralgia. Neuroradiology 1995;37:353-5

4. Arthur MG. Improved visualization of the foramen ovale for percutaneous approaches to the gasserian ganglion $\mathrm{J}$ Neurosurg 1994;80:156-9

5. Barker FG, Jennatta PJ, Bissonette DJ, Larkins MV, Jho HD. The long-term outcome microvascular decompression for trigeminal neuralgia. N Engl J Med 1996; 334:1077-83

6. Cho DY, Chang CGS, Wang YC. Repeat operations in failed microvascular decompression for trigeminal neuralgia. Neurosurgery 1994;35: 665-70

7. Janetta PJ, Marc RM, Brent LC. Microvascular decompression of the trigeminal nerves. J Neurosurg 1999;90: $1-8$

8. Jannetta PJ. Microvascular decompression of the trigeminal nevre for tic douloreux, in Youmans-Neurological Surgery.Saunders Company, Fourth edition, 1996, volum 5 pages 3404-15

9. Sunn HL, Elad IL, Alan MS, Amin K. Recurrent trigeminal neuralgia attributable to veins after microvasculer decompresyon Neurosurgery 2000;46: 356-62

10. Cruccu G. Idiopathic and symptomatic trigeminal pain. J Neurol Neurosurg Psychiatry 1990; 53: 1034-1042
11. Ishwar CP, M.Ch, Moss T. Tefloninduced granuloma following treatment of trigeminal neuralgia by microvascular decompression. Neurosurg 1997;87:454-7

12. Kim JB, Konstantin VS. On the natural history of trigeminal neuralgia. Neurosurgery 2000;46:152-5

13. Sjaastad O, Pareja JA, Zukerman JJ, Kruszewski P. Trigeminal neuralgia clinical manifestations of first division involvement. Headache1997:346-57

14. Zakrzewska J lopez BC.New techniques for surgical management of trigeminal neuralgia. Pain 2004;109:520

15. Gass A, Kitchen N, MacManus DG, Moseley IF, Hennerici MG, Miller DH, Trigeminal neuvralgia in patients with multiple sclerosis lesion localization with magnetic resonance imaging. Neurology 1997;49(4):1142-4

16. Young RF, Vermeulen SS, Grimm P, Blasko J, Posewith A. Gamma knife radio-surgery for treatment of trigeminal neuralgia idiopathic and tumor related. Neurology 1997;48(3):608-12

17. Brismann R. Gamma knife surgery with a dose of 75 to 76.8 gray for trigeminal neuralgia. J Neurosurg 2004;100:447-50

18. Waidhauser E, Steude U. Evaluation of patients wiyh atypical trigeminal neuralgia for permanent electrode implant by test stimulation of the ganglion gasseri. Stereotact Func Neurosurg 1994;62:3048

19. Wentz KU, Röther J, Scwartz A, Mattle HP, Edelman RR. Intracranial vertebrobasilar system MR angiography. Radiology 1994; 190:105-10

20. Burchiel KJ. Trigeminal neuropathic pain. Acta Neurochir Supp 1993;58:145-49 